\title{
Mobile Agent Based Project Management System
}

\author{
Nuo Zhang ${ }^{1}$, Fuxiang Zhang ${ }^{2 *}$, Rongting $\mathrm{Li}^{2}$, Yuejing $\mathrm{Zhao}^{2}$ \\ ${ }^{1}$ Department of Economy and Trade, Shijiazhuang University of applied Technology, Shijiazhuang, \\ Hebei, P. R. China \\ ${ }^{2}$ School of Mechanical Engineering, Hebei University of Science and Technology, Shijiazhuang, Hebei, \\ P. R. China \\ *Corresponding Author.
}

\begin{abstract}
Scientific and technological schedule makes the products' life cycles be becoming shorter. The companies should make product research and putting into the market more rapidly. Product rapid development and popularization depends on project management, and project schedule optimization is the core of project management. The structure of the project schedule optimization was detailed analysis and mobile agent was import in project schedule optimization system. The event model of mobile agent, the moving process, the model of aglet and the implement method of mobile agent in project schedule optimization system were introduced. This analysis shows the mobile agent the internal structure and negotiation algorithm abundantly. It will play a guiding role for the development of the whole project schedule optimization system and the application of the mobile agent in the project schedule optimization system.
\end{abstract}

Keywords: Mobile agent, Project schedule, Architecture, Aglet

\section{Background}

Project management is the core content of the schedule management. In order to effectively control the project time, resources and costs of the process, the project schedule must be optimized throughout the entire process of project management [1]. Schedule management covers the organization and quality assurance of the project implementation process. It includes the allocation of project factors of production, but also reflects the continuity of project implementation and balance. The preparation of the schedule plan is an important part of the project management activities. It is already forming a complete system of preparation and preparation tools. Therefore, how to prepare the scientific schedule plan are great significance for the construction and the benefits of the project. The popularization of computer technology makes the project management information system widely used. With the agent technology in network management, e-commerce, workflow and business process management and other fields are more and more widely used. To introduce agents into project management is of great practical significance [2].

With the development of computer technology, agent and multi-agent system become the hotspot of research [3, 4]. Agent technology is suitable for dealing with complex, cooperative and unpredictable problems. It solves the problem of information resource sharing and effective use of expert knowledge, and can realize the simultaneous operation and interaction of multiple systems. Multi-agent system provides decentralized control and parallel processing, which can be quickly solved the problem [5]. Multi-agent system can solve different problems through the combination of multiple agents. Agent for different problems can be modularized, thus enhancing the system maintainability. Mobile Agent is characterized by mobility, which can move and run autonomously among different machines in a heterogeneous network environment.

The project portfolio selection problem (PPSP) has been studied during the last decade. Most studies on PPSP generally analyze the internal relationship between project portfolio selection and project scheduling. Taking the

ISSN: 0010-8189

(c) CONVERTER 2021

www.converter-magazine.info 
scheduling of project activities as a sub-problem of portfolio selection can help improve the overall performance of the organization, even if it increases the complexity of decision-making [6]. The project scheduling problem is a typical NP-hard problem. Generally, artificial intelligence solutions for scheduling problems include heuristic algorithms, intelligent optimization algorithms, and MAS-based methods [7]. Many algorithms have been used to solve the problem of project scheduling optimization. Among all heuristic algorithms, Lagrangian relaxation algorithm performs well in scheduling problems due to its high efficiency in obtaining high-quality resolution [8]. The performance of intelligent optimization algorithms in terms of speed and effectiveness is outstanding in finding the best solution to project management problems [9, 10]. Through the combination of the concept of collaborative management and modern optimization technology, they achieve the multi-objective collaborative management and reasonable allocation and use of construction project resources.

Although these studies have obtained a large number of methods to solve the scheduling problem, they cannot completely provide a system solution for the optimization of project scheduling based on mobile agents.

\section{System architecture}

\subsection{Agent architecture}

Figure 1 shows the mobile agent-based project management system structure. In the Agent platform A, the project managers use the Project Decomposition Agent, the Task Assignment Agent and the Optimization Control Agent realize the management operation and get the Process Agent and the Experience Agent. The project staff works on the Agent platform B to generate the Process Agent and the Experience Agent. All the Process Agent and the Experience Agent can achieve two-way data exchange with the Database server.

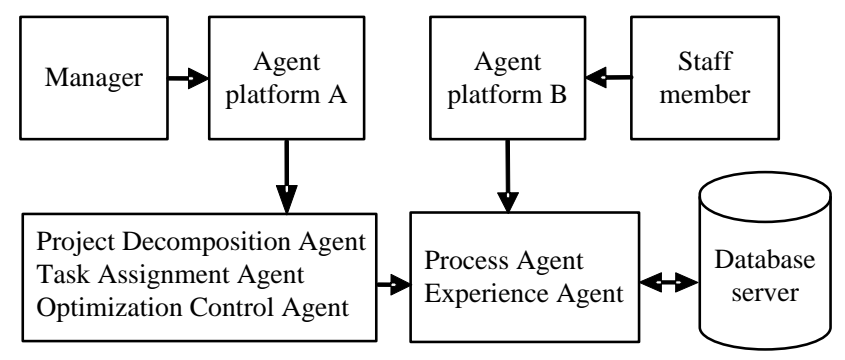

Figure 1 Project scheduling optimization system architecture based on mobile agent

Figure 2 shows multi-agent architecture of the project scheduling optimization system based on mobile agents. The agents in the system include the Project Decomposition Agent, the Task Assignment Agent, the Process Agent, the Optimization Control Agent, the Quota Agent and the Experience Agent.

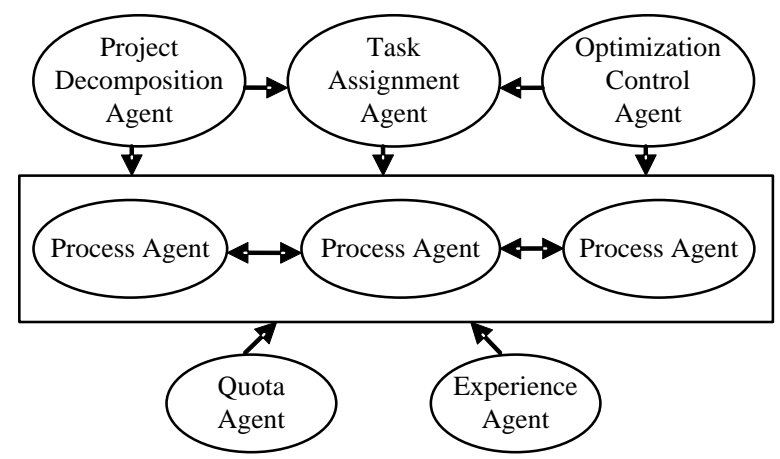

Figure 2 The multi-agent architecture of the project management system

ISSN: 0010-8189

C CONVERTER 2021

Www.converter-magazine.info 


\subsection{Project Decomposition Agent}

The Project Decomposition Agent has the knowledge of WBS. It decomposes the project from top to bottom layer by layer, and decomposes the whole task from the project task to each specific work process according to the level, and forms a work breakdown structure model. It works as follows: After inputting the project information in the computer terminal, the project manager automatically generates the auxiliary project of the Project Decomposition Agent and interacts with the system. The agent identifies the state information of the project through the perception, and then the internal decision module decomposes the project. Figure 3 shows the internal structure of the Project Decomposition Agent and its communication relationship.

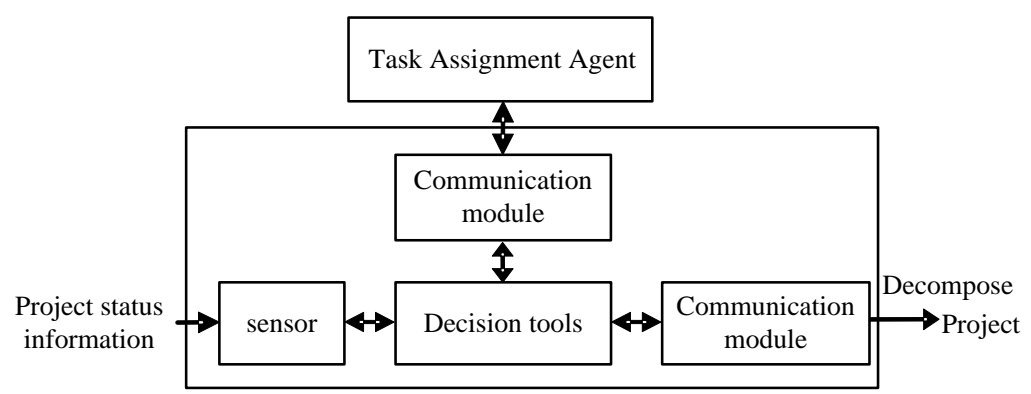

Figure 3 The internal structure of Project Decomposition Agent and its communication relationship

\subsection{Task Assignment Agent}

Task Assignment Agent itself has the knowledge of PERT/CPM. It divides the process activities of the project decomposition into different agents, that is, different agents to represent the various processes, and the agent itself contains the project logic relations. It works as follows: After decomposing the project, the system automatically generates the Task Distribution Agent to communicate with the Project Decomposition Agent to get the information of each process after decomposition. Meanwhile, the process constraint is also taken into account to allocate the process. The Task Assignment Agent interacts with the system and creates a Process Agent, assigning each Process Agent to the work machine for work. Figure 4 shows the internal structure of the Task Assignment Agent and its communication relationship.

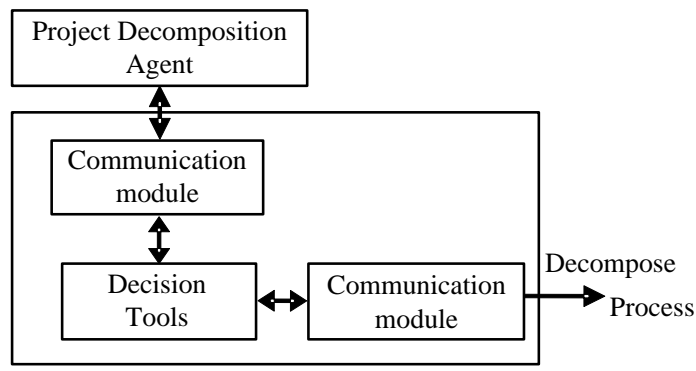

Figure 4 The internal structure of the Task Assignment Agent and its communication relationship

\subsection{Quota Agent}

Quota Agent contains the quota library; the quota library reflects the average level of social production. Quota Agent's personality analysis tools are provided by the Process Agent. The Quata Agent is connected with the Process Agent, the Control Agent and the Experience Agent to obtain the exact information of the existing process. By querying the quorum library, the Quota Agent compare and match the personality analysis tool with the knowledge to obtain the relevant data of each process in the project and to analyze the result to the Process Agent, and to guide the calculation of the duration of the Process Agent. Figure 5 shows the internal structure of the Quota Agent and its communication relationship.

ISSN: 0010-8189

(C) CONVERTER 2021

www.converter-magazine.info 


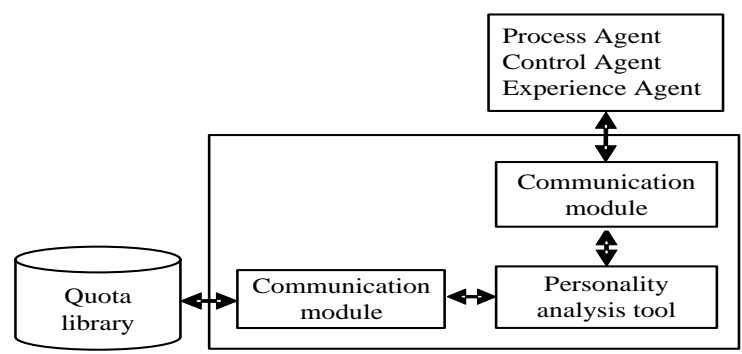

Figure 5 The internal structure of the Task Assignment Agent and its communication relationship

\subsection{Experience Agent}

An Experience Agent contains the similar work of the previous case information, such as duration, number of workers, materials used, costs, and so on. Experience is a summary of a great deal of work practice. As an expert, an Experiential Agent can provide great support and help for project control and decision-making. The working process is mainly to communicate with the Process Agent and the Optimization Control Agent, get the basic and individual information of the existing project, compare with the database information, and pass reasonable data to the Process Agent. Figure 6 shows the internal structure of the Experience Agent and its communication relationship.

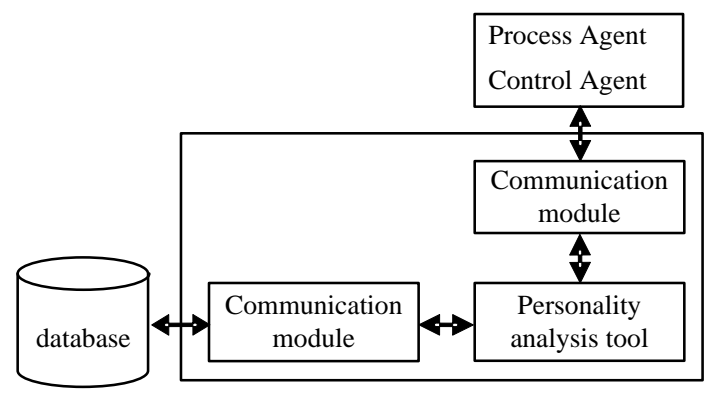

Figure 6 The internal structure of the Experience Agent and its communication relationship

\subsection{Optimization Control Agent}

Initially generated duration plan is usually not in line with project duration, cost and other aspects of the constraints, it must be further optimized. It mainly includes: time optimization, cost optimization, and resource optimization. This paper mainly study the time optimization. It works as follows: First, at the computer terminal of the project manager, the Optimization Control Agent obtains the planned duration from the other end, and the decision-making tool is responsible for the comparison of the construction period and the contract duration. Time requirements and the task of each agent in the system are completed. If not meet the requirements, the Optimize Control Agent needs to return the unqualified information to the Process Agent in consideration of various constraints. The Process Agent with the duration requirements and programs were re-assigned to the process machine for duration calculation. This process is repeated until the duration of the contract is met. Figure 7 shows the internal structure of the Optimal Control Agent and its communication relationship.

ISSN: 0010-8189 


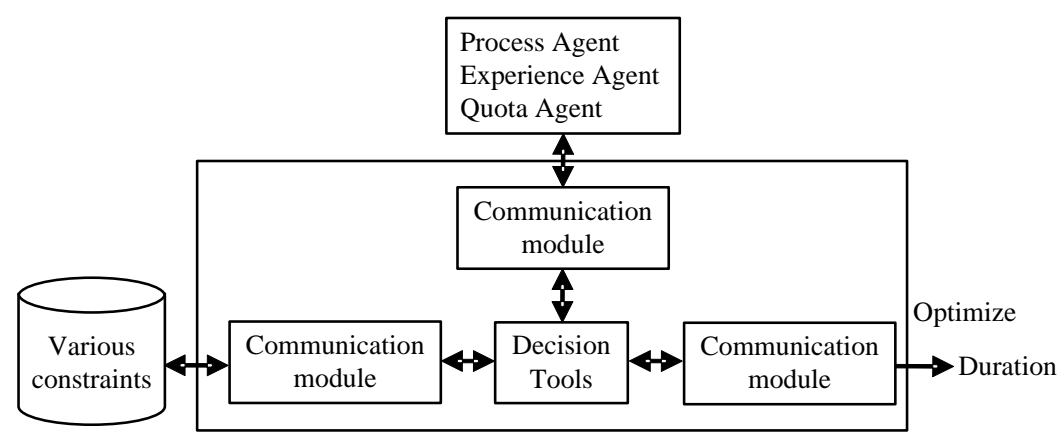

Figure 7 The internal structure of the Optimal Control Agent and its communication relationship

\subsection{Process Agent}

The Process Agent can automatically negotiate with other agents to acquire enough knowledge to calculate the duration, cost of resources and costs. The purpose of each Process Agent is to minimize the costs. Its main task is to calculate the time limit and feedback information to the Optimization Control Agent. When the duration needs to be adjusted, the appropriate Process Agent can be selected by the algorithm set by the system. Figure 8 shows the internal structure of the Process Agent and its communication relationship.

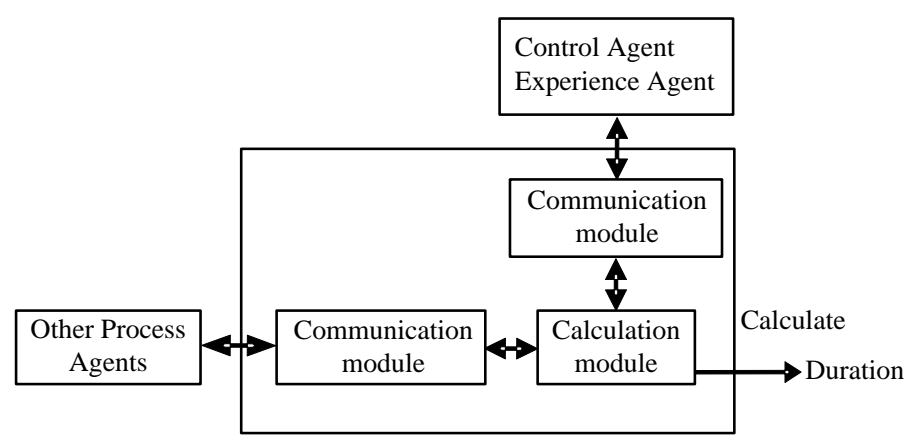

Figure 8 The internal structure of the Process Agent and its communication relationship

\section{Realization of the mobile agent system in project scheduling optimization}

\subsection{Event Model of the Agent}

Aglets is a Java-based mobile agent system developed by IBM. The source code and documentation are completely open to the public, making it ideal for learning and developing applications. Aglets is an Applet object, with agent behavior, and also has the characteristics of agent and Applet object. Aglet is a mobile agent. Aglets system provides a visual proxy service interface Tahiti, can be moved between the machines installed Tahiti, can also be moved between the different Tahiti servers of the one machine [11]. Figure 9 shows an Aglet event model. 


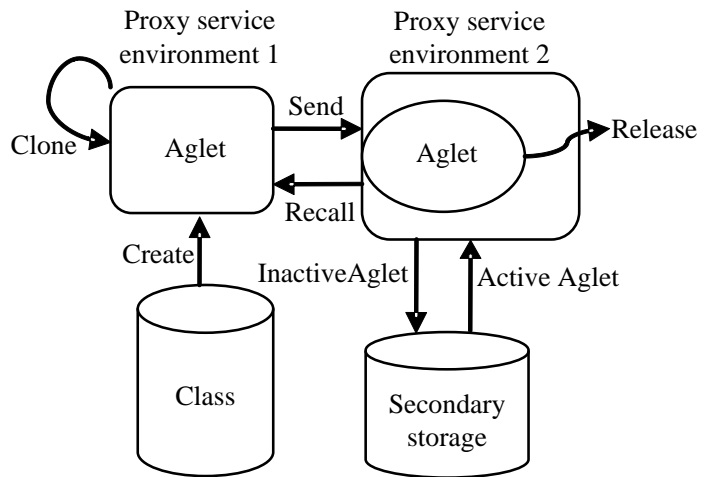

Figure 9 An Aglet event model

\subsection{Event Model of the Agent}

Move can be divided into two types: strong mobile and weak mobile. A moveable process consists of three segments: code segment, data segment, and execution segment. A strong move consists of a movement of code segment, data segment, and execution segment, while a weak movement contains only movement of code segment and data segment. Aglets is a weak mobile system, the requirements of the target machine is able to run code independently, and can simply move. So that the amount of data transmitted less, the operation cost less, it is high efficiency. The lines and conditions of the Aglet moves are included in the task code. Figure 10 shows the Aglet movement process.

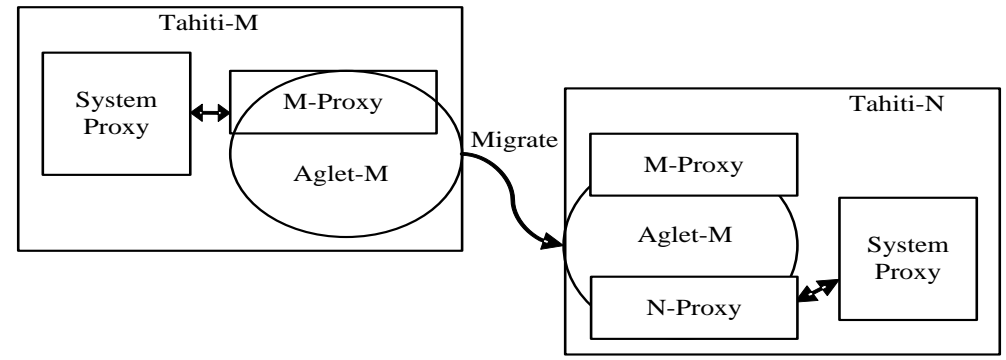

Figure 10 The Aglet movement process

The codes of Client Aglets send email-Aglet are as follows:

AgletContext context=getAgletContext () ;

AgletProxy proxy=context.createmailAglet (null. "example" .getProxy());

URL url=new URL ();

remoteProxy=proxy.dispatch(url);

\subsection{Aglet Model in the Project Scheduling Optimization System}

The mobility of the Aglet can be realization by dividing the supported environments into three layers: Aglet Proxy, Agent Transfer Protocol and Aglet Context. Figure 11 shows the Aglet object model.

ISSN: 0010-8189 


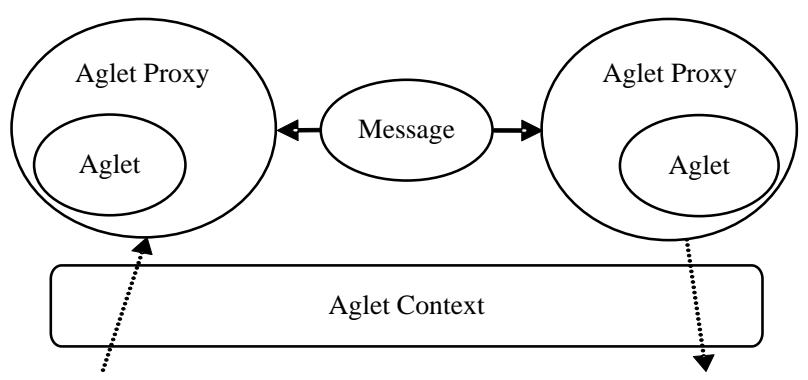

Figure 11 The Aglet object model

The security protection that the Aglet achieved is one-way. The Aglet focus on the system protection, and the protection of the Aglet itself is lack. Aglet internal events use the delegation model of the Java system, and use ATP as communication languages between the Aglets. Figure 12 shows the Aglet communication model.

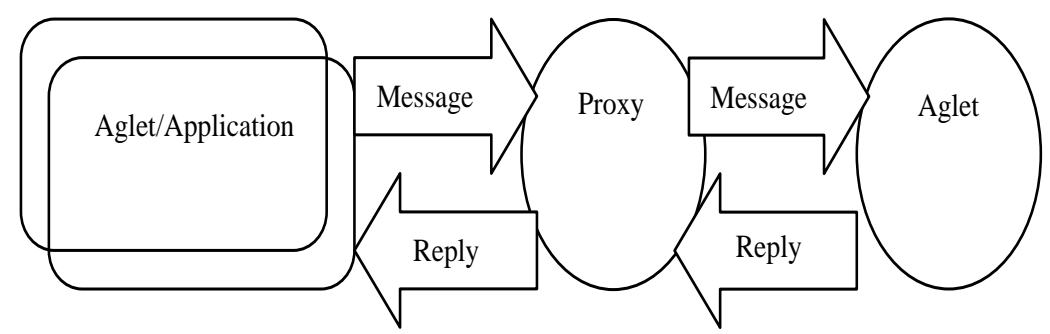

Figure 12 The Aglet communication model

\section{Conclusions}

According to the technical characteristics of the current project schedule optimization, mobile agent-based novel coronavirus epidemic management system architecture is proposed. The mobile agent is introduced and the internal structure of the mobile agent is designed. On the Aglet platform, the codes of the agent internal structure are programmed and optimized, and the negotiation algorithm is realized. The development of the whole system is completed. The system makes use of the characteristics of the mobile agent in the process of project schedule optimization.

\section{Acknowledgements}

This work is supported by National Key R \& D Plan Project of China (No. 2018YFB1308700), Hebei Province Science and Technology Project (No. 19011826Z), Shijiazhuang Science and Technology Project (No. 201080021A), and Hebei University of Science and Technology Scientific Research Fund.

\section{References}

[1] H. Hammouch, H. Medromi, A. Sayouti, "Toward an intelligent system for project management based on the multi agents systems," Proc. of 10th International Conference on Intelligent Systems. Pp.1-6, 2015.

[2] F. Fu, H. Zhou, "A combined multi-agent system for distributed multi-project scheduling problems," Applied Soft Computing, 107402, 2021.

[3] L. Qiu, K. Li, “The research of intelligent agent system architecture based on cloud computing. Proc. of 12th International Conference on Computational Intelligence and Security (CIS),” pp. 693-696, 2016.

[4] M. Sharma, M. Firdaus, R. K. Chatterjee, et al., "Constraint specification in multi agent system," Proc. of IEEE Region 10 Conference (TENCON), pp. 2404-2409, 2016.

[5] Q. Chen, "Research on cloud computing resource management model based on multi-agent system.

ISSN: 0010-8189

(C) CONVERTER 2021 
Proc. of 12th International Conference on Computational Intelligence and Security (CIS)," pp. 378-381, 2016.

[6] Y.Y. Shou, W.W. Xiang, Y. Li, et al., "A multiagent evolutionary algorithm for the resource-constrained project portfolio selection and scheduling problem," Mathematical Problems in Engineering, pp. 1-9, 2014.

[7] J.H. Li, L.Y. Li, B.X Yang, et al., "Development of a collaborative scheduling system of offshore platform project based on multiagent technology," Advances in Mechanical Engineering, pp. 1-15, 2014.

[8] F. Jarray, "A lagrangean-based heuristics for the target covering problem in wireless sensor network.," Applied Mathematical Modelling, vol. 37, no. 10-11, pp. 6780-6785, 2013.

[9] S. Chernov, L. Chernova, L. Chernova, et al., "The analysis of gomory algorithm convergence in integer linear optimization problem: projects management," 2019 IEEE 14th International Conference on Computer Sciences and Information Technologies (CSIT), 2019.

[10] A.V. Goryachev, N.E. Novakova, "The project optimization with the use of heuristic algorithms," 2017 XX IEEE International Conference on Soft Computing and Measurements (SCM), 2017.

[11] G. Karjoth, D.B. Lange, M. Oshima, “A security model for Aglets. IEEE Internet Computing,” vol. 1, pp. 68-77, 1997. 